\title{
Análisis Multifactorial Del Consumo Energético de un Proceso de Fresado Frontal
}

Luis M. Sarache, Ramón Ramírez y Víctor M. Guédez

Universidad de Los Andes, Facultad de Ingeniería, Escuela de Ingeniería Mecánica, Grupo de Tecnología GRUTEC, Postgrado de Ingeniería en Mantenimiento, Mérida. Edo. Mérida-Venezuela (e mail:sarachel@ula.ve)

\begin{abstract}
Resumen
Este trabajo muestra una evaluación y análisis multifactorial sobre el consumo energético de piezas fresadas utilizando un Diseño Experimental Factorial. Se consideran las variables profundidad de corte, velocidad de avance y uso de fluido de corte. Los datos se obtuvieron mediante una tarjeta de adquisición de datos y los cálculos sobre significancia se efectuó con la utilización del software SEMPRO II. Además de la calidad de las piezas, se analiza el uso racional de la energía, ya que el costo de producción de una pieza es afectado por la potencia consumida. Se concluye que con el uso del refrigerante o fluido de corte disminuye el gasto energético en un $28 \%$, cuando se trabaja con mínima profundidad y velocidad de avance de la pieza, lo que hace necesario mejorar los diseños de piezas a mecanizar, orientadas al mínimo de desperdicio que se logra con profundidades muy pequeñas.
\end{abstract}

Palabras claves: corte de metal, diseño factorial, análisis multifactorial, uso de energía

\section{Multifactorial Analysis of the Energetic Consumption of a Process Face Milling}

\begin{abstract}
This work shows an evaluation and multifactorial analysis on the energetic consumption of pieces face milling using an Experimental Design Factorial, are considered to be the variables depth of cut, feed per tooth and the use or not of Cutting Fluid. The values generated of the byline it was achieved by means of a information acquisition card and the calculations on significance was effected(carried out) by the utilization of the software SEMPRO 2, that allows the analysis multifactorial, the surface of response and the equation of variable. It is important to give a sense different from the efficiency of the processes of manufacture that affect the quality of the pieces, though the quality is important for reasons of competitiveness, also and must be the rational use of the energy, since the cost of production of a piece is affected by this one (power). One concludes that the use of cooling or cutting fluid diminishes the energetic expense in $28 \%$, when one works with minimal depth and advance of the piece, which it makes necessary to improve the designs of pieces to mechanizing, orientated to the minimum waste that is achieved by very small depths.
\end{abstract}

Keywords: metal cutting, factorial design, multifactorial analysis, evaluation of power 


\section{INTRODUCCION}

En la actualidad, es vital conocer el efecto de las variables características de los procesos de mecanizado que afectan el consumo de energía, ya que éste determina en buena medida los costos asociados a la producción que tienen importancia por ser consumos energéticos en un mundo donde la energía y la utilización de los recursos se hace cada día más importante, por lo anterior el estudio es aplicado a un proceso de planeado con una fresa frontal, ya que la mayoría de los centros de mecanizados utilizan esta operación como básica para cualquier proceso, de allí la importancia de determinar y controlar algunas variables que siendo significativas, permitan un uso racional de consumo de energía. Como variables de estudio se tienen la profundidad de corte, el uso de refrigerante y el avance de la pieza respecto a la herramienta; se pretende obtener un patrón de respuesta que permita indicar cuáles inciden significativamente, utilizando para ello, la metodología y software del Sistema de Evaluación Multifactorial de Procesos, Productos y Organización SEMPRO 2, desarrollado por (Sarache y Peña 2004).

Para el estudio y posterior análisis de las respuestas de las variables, se asignan para cumplir con la metodología, dos niveles a cada una de ellas, lo que implica realizar 8 ensayos básicos y 8 replicas, siendo un total de 16 ensayos para definir las tendencias. Se preparan las piezas de trabajo, que consisten en barras de sección transversal cuadrada de $25 \mathrm{~mm}$ de acero ASTM-A36 de Resistencia a la Tracción 400 Mpa y de 100 mm de longitud y se efectúan las pruebas bajo las distintas condiciones según el Diseño Factorial Básico (DFB), utilizando una tarjeta de adquisición de datos de la National Instruments, siguiendo planteamientos dado por Aoyama y Ameraiya (2000) sobre el uso de sensores para la detección de fuerzas de corte, utilizando conexiones particulares sin considerar el efecto Villari, tal que garantice la generación de una gran cantidad de data con alta validez y disminución de los errores, ya que para una operación de fresado hay números infinitos de contratos de la herramienta y la instrumentación tiene que ser factible de generar esta recolección de datos como lo plantea (Chu, 1999) para que sean confiables.

Se demuestra en la experimentación que al trabajar con el mínimo valor de profundidad de $1 \mathrm{~mm}$., avance de $56 \mathrm{~mm} . / \mathrm{min}$., y con el uso de refrigerante, se obtiene el menor consumo de potencia y por ende de energía, siendo ésta la situación más favorable y la menos favorable, se obtiene al combinar la mayor profundidad con $2 \mathrm{~mm}$., avance de 90 $\mathrm{mm} / \mathrm{min}$., y sin el empleo de refrigerante, se trató de seguir las pautas metodológicas de Chern y Dornfeld (1996), aun cuando su trabajo se basa en la formación de rebabas en el fresado, donde el manejo correcto de la data adquirida es fundamental.

\section{FASE PRE-EXPERIMENTAL}

En el fresado, el movimiento principal de corte está a cargo de la herramienta, de modo que, el desplazamiento de alimentación es dado por la mesa de fresar a la pieza, donde cada uno de los dientes de la herramienta corta una viruta en forma de cuña, entonces, el espesor de la viruta pasa de un valor mínimo a un valor máximo en el proceso y que dependerá: del movimiento de alimentación, del diámetro de la fresa utilizada y de la profundidad de corte. Esta característica del trabajo basado en los dientes o sus ángulos de filo, sometidos a esfuerzos variables producto del cambio de un mínimo a un máximo de las fuerzas actuantes, ha cobrado mucho interés por estar asociadas a la generación de superficies de ultra o nanoprecisión (Schey, 2000), donde la evaluación de la potencia consumida no puede tratarse de manera aislada, sino asociada a la combinación de factores que inciden en los gastos energéticos.

Con el experimento se determina el nivel de significancias de las variables en la potencia requerida para el planeado de una pieza. Se aplica el Diseño Factorial Básico (DFB) para mejorar el control de del proceso, logrando que sea afectado con la menor cantidad posible por fuentes de variabilidad externa. La metodología estadística enfoca el análisis de un problema que involucre datos sujetos a errores experimentales y tratará de cubrir los dos aspectos en cualquier problema experimental, como son el diseño del experimento y el análisis de resultados, Sarache (2001) y Montgomery (1996) explican que estos dos temas están estrechamente relacionados, de allí que se aplica la técnica de Ishikawa y del Grupo Nominal, para reducir el universo de variable 
a solo tres: velocidad de avance, profundidad de corte y uso de refrigerante, debido a que algunas variables son dependientes del material como tipo y geometría de la pieza, otras dependientes de la herramienta como geometría del inserto y calidad y otras variables de la máquina, como sistemas de sujeción tanto de la pieza como de la herramienta, todas ellas constantes para un DFB $2^{3}$.

Las variables e evaluar son: profundidad de corte, velocidad de avance y el uso o no de refrigerante con relación agua - aceite soluble $2: 1$. Los valores de los niveles de las variables cuantitativas velocidad $y$ profundidad están dentro de los rangos sugeridos por (Casillas, 1982) y (Krar y Check, 2002), se seleccionan: profundidad $\left(P_{C}\right)$ entre 1 y $2 \mathrm{~mm}$. rango $(1-2) \mathrm{mm}$., avance por diente $\left(\mathrm{S}_{\mathrm{Z}}\right): 0.05$ y 0.15 $\mathrm{mm}$./diente. rango $(0.05-0.15) \mathrm{mm} /$ diente y velocidad $\left(V_{C}\right)$ entre 40 y $60 \mathrm{~m} / \mathrm{min}$., rango equivalente $(0.66-1.00) \mathrm{m} / \mathrm{s}$.

Se recalculan los valores de velocidad de rotación y avances para ajustarlos a los disponibles en la Fresadora Marca JAROCIN, Tipo: FWD32J del Laboratorio de Tecnología Mecánica de la Facultad de Ingeniería de la Universidad de Los Andes, para cumplir con las especificaciones.

Tabla 1. Factores y Niveles de las variables del Diseño Factorial Básico (DFB $2^{3}$ ).

\begin{tabular}{|c|c|c|}
\hline $\begin{array}{c}\text { Factores o Variables } \\
\text { de estudio }\end{array}$ & $\begin{array}{c}\text { Nivel } \\
\text { bajo } \\
(-)\end{array}$ & $\begin{array}{c}\text { Nivel } \\
\text { alto } \\
(+)\end{array}$ \\
\hline [V] Avance de pieza $(\mathrm{mm} / \mathrm{rev}) \mathrm{X}_{1}$ & 56 & 90 \\
\hline [P] Profundidad de corte $(\mathrm{mm}) \mathrm{X}_{2}$ & 1.00 & 2.00 \\
\hline [R] Refrigerante (adim.) & $\mathrm{Si}$ & No \\
\hline
\end{tabular}

Se utilizó una fresa universal de insertos marca JELENIA GORA, de especificaciones hR 257.1-080 de seis insertos de Carburo de Tungsteno, de especificaciones SNUN 120307.

Velocidad de avance para nivel mínimo $\mathrm{Vc}=$ $0.66 \mathrm{~m} / \mathrm{s}$ que corresponde $\mathrm{N}=159.15 \mathrm{rpm}$. y el valor de rpm. más cercano disponible de la fresadora es $180 \mathrm{rpm}$. equivalente a un avance de mesa disponible de $56 \mathrm{~mm} . / \mathrm{min}$.

Velocidad de avance para nivel máximo $\mathrm{Vc}=$ $1 \mathrm{~m} / \mathrm{s}$ que corresponde $\mathrm{N}=238.73 \mathrm{rpm}$. y el valor de rpm. más cercano disponible de la fresadora $280 \mathrm{rpm}$. equivalente a un avance de mesa de $90 \mathrm{~mm} . / \mathrm{min}$.

Con estos valores de velocidad de avance se obtienen valores similares de avance por diente como es lo deseado en la presente investigación, de $0,052 \mathrm{~mm}$./diente para el primer caso de $56 \mathrm{~mm} . / \mathrm{min}$. y de 0,053 $\mathrm{mm}$./diente para el segundo de $90 \mathrm{~mm} . / \mathrm{min}$., tal como aparecen en la Tabla 1.

\section{FASE EXPERIMENTAL}

Hubo la necesidad de utilizar una tarjeta de adquisición de datos de la National Instruments DAQPAD6020 USB, para obtener los valores de Voltaje (Voltios) e Intensidad de Corriente (Amperios) por fase en cada una de las tres fases a la entrada del motor. La tarjeta se configuró para tomar datos a un intervalo de $740 \mu \mathrm{s}$, el cual permitió calcular la potencia consumida por el motor en las diferentes configuraciones a las que fue sometido el proceso casi como variable continua. Una vez tomados los datos y procesados su análisis según (Gutiérrez y De la Vara, 2003), los valores obtenidos se muestran en la Tabla 2. Para el cálculo del efecto de cada variable independiente o en interacción se emplea la ecuación planteada por Box et al. (1978):

Tabla 2. Resultados Experimentales de la evaluación de potencia en fresado aplicando un Diseño Factorial Básico (DFB $2^{3}$ )

\begin{tabular}{|c|c|c|c|c|c|c|}
\hline \multirow[t]{2}{*}{ Orden } & \multicolumn{3}{|c|}{ Variable } & \multicolumn{3}{|c|}{$\begin{array}{c}\text { Potencia Consumida (W) } \\
\text { Corridas }\end{array}$} \\
\hline & $\mathrm{P}$ & $\mathrm{R}$ & V & 1 & 2 & Media \\
\hline (2) & - & - & - & & 170,7 & \\
\hline (13) & & & & 162,91 & 6 & 166,84 \\
\hline $\begin{array}{c}(5) \\
(11)\end{array}$ & + & - & - & 187,97 & $\begin{array}{c}188,1 \\
5\end{array}$ & 188,06 \\
\hline (8) & - & + & - & & 172,2 & \\
\hline (15) & & & & 175,62 & 9 & 173,96 \\
\hline $\begin{array}{l}(1) \\
(14)\end{array}$ & + & + & - & 205,50 & $\begin{array}{c}204,2 \\
3\end{array}$ & 204,87 \\
\hline $\begin{array}{c}(6) \\
(16)\end{array}$ & - & - & + & 169,73 & $\begin{array}{c}172,2 \\
2\end{array}$ & 170,98 \\
\hline $\begin{array}{l}(7) \\
(9)\end{array}$ & + & - & + & 234,68 & $\begin{array}{c}217,9 \\
8\end{array}$ & 226,33 \\
\hline $\begin{array}{l}(3) \\
(10)\end{array}$ & - & + & + & 210,95 & $\begin{array}{c}206,7 \\
9\end{array}$ & 208,87 \\
\hline (4) & + & + & + & & 261,3 & \\
\hline (12) & & & & 252,77 & 7 & 257,07 \\
\hline
\end{tabular}


Efecto $[\mathrm{x}]=\frac{2}{\mathrm{n}}\left[\begin{array}{l}(\text { Valor medido }) \times \\ (\text { Signo }+/- \text { de la columna })\end{array}\right]$

A manera ilustrativa se calcula el efecto de la variable $[P]$ profundidad de corte $(\mathrm{mm})$.

Efecto $_{[1]}=\quad[-166,84+188,06-173,96+204,87-$ $170,98+226,33-208,87+257,07] / 4=38,9 \mathrm{~W}$

Es necesario definir si el efecto de la variable en estudio es "significativo", con lo cual se procede a separar los efectos principales de los de interacción de dos o más variables, empleando los niveles de significancia de las variables, que mediante el cálculo del error permite determinar cual será la desviación permisible, siempre y cuando su valor sea el doble del error. Para esto, se plantea la siguiente ecuación:

$\mathrm{S}^{2}=\sum_{\mathrm{i}=1}^{\mathrm{n}} \frac{\left[\text { Xprom }_{\mathrm{i}}-\bar{X}_{\text {prom }}\right]^{2}}{2 \mathrm{~g}}$

Para la significancia se calcula la desviación permisible, en este caso se realizaron dos réplicas de cada prueba, por lo que para calcular la varianza del efecto de experimentación $\left[\operatorname{Var}_{(\mathrm{x})}\right]$ y con ella la desviación estándar $[\sigma]$ como su raíz, se emplea la siguiente ecuación:

$\operatorname{Var}_{(x)}=\left(\frac{1}{2}\right) S^{2}$

$\sigma=\sqrt{\operatorname{Var}_{(\mathrm{x})}}$

Tabla 3. Resultados de los efectos individuales y de interacción del consumo de potencia.

\begin{tabular}{cccc}
\hline Efectos & $\begin{array}{c}\text { Valor Estimado } \\
(\mathrm{W})\end{array}$ & \multicolumn{2}{c}{$\begin{array}{c}\text { Error } \\
\text { Estándar }\end{array}$} \\
\hline \multicolumn{4}{c}{ Efectos de variables } \\
\hline$[\mathrm{P}]$ & 38,92 & \pm & 5,31 \\
{$[\mathrm{R}]$} & 23.14 & \pm & 5,31 \\
{$[\mathrm{~V}]$} & 32,38 & \pm & 5,31 \\
\hline Efectos de & Interacción de dos variables \\
\hline$[\mathrm{P}][\mathrm{R}]$ & 0,63 & \pm & 5,31 \\
{$[\mathrm{P}][\mathrm{V}]$} & 12,91 & \pm & 5,31 \\
{$[\mathrm{R}][\mathrm{V}]$} & 11,18 & \pm & 5,31 \\
\hline
\end{tabular}

Efectos de Interacción de tres variables

$[\mathrm{P}][\mathrm{R}][\mathrm{V}] \quad-04,21 \quad \pm \quad 5,31$

\section{FASE POST-EXPERIMENTAL}

Con los valores presentes en la Tabla 3 , se puede compara el efecto de las variables con la desviación presente en el experimento y de allí se determinan los valores a considerar significativos al ser superiores a dos veces el valor de la desviación, lo cual asegura una confiabilidad del $96 \%$ en el análisis, que se evidencia con el valor experimental obtenido de la variable y la hace significativa para el estudio.

\section{DISCUSION}

Una vez efectuado los cálculos respectivos y tabulados, se hace necesario orientar el estudio, en primer lugar, a las condiciones en el proceso de planeado y los niveles de significancia de las variables, tanto individuales como de interacción $y$, en segundo lugar, basado en las características del DFB, las tendencias de los factores involucrados mediante la aplicación del diagrama de dos vías como elemento de análisis.

Como se puede apreciar en la figura 1, cuando la velocidad de avance decrece, asociada a una menor profundidad, disminuye el consumo de potencia, generando un decrecimiento de $71.31 \mathrm{~W}$ equivalente a un $29,5 \%$ en relación con el punto de operación más desfavorable, resultado de una baja velocidad de avance y baja profundidad de corte. Cuando se incrementa la velocidad de avance, sin modificar la profundidad de corte se produce una mejora de $51,78 \mathrm{~W}$ o un $21,42 \%$ en relación con el punto de operación más desfavorable; pero, si se aumenta la profundidad de corte sin modificar la velocidad de avance, se produce una mejora de $45,23 \mathrm{~W}$ o un $18,71 \%$ en relación con el punto de operación más desfavorable. Por lo tanto, el menor consumo de potencia se logra a la velocidad de avance y profundidad de corte más baja; para valores centrales de: 192.89, 193.49 y $195.05 \mathrm{~W}$, se consigue la ecuación dada por el SEMPRO II:

Potencia $=197.13+19.46 X_{1}+11.57 X_{3}$

Según la figura 2, en la medida que la velocidad de avance decrece, usando el refrigerante se disminuye el consumo de potencia, generando un decrecimiento de $55,53 \mathrm{~W}$ o un $28,84 \%$ en relación con el punto 
de operación más desfavorable. Cuando se incrementa la velocidad de avance, usando refrigerante se produce una mejora de 34,32 W o un $14,73 \%$ en relación con el punto de operación más desfavorable; pero, si no se usa refrigerante a la velocidad de avance menor, se produce una mejora de $43,56 \mathrm{~W}$ o un $18,69 \%$ en relación con el punto de operación más desfavorable. Por lo tanto el menor consumo de potencia se logra a la velocidad de avance baja y usando refrigerante; para valores centrales de: 183.75, 182.24 y $186.31 \mathrm{~W}$, se consigue la ecuación dada por el SEMPRO II:

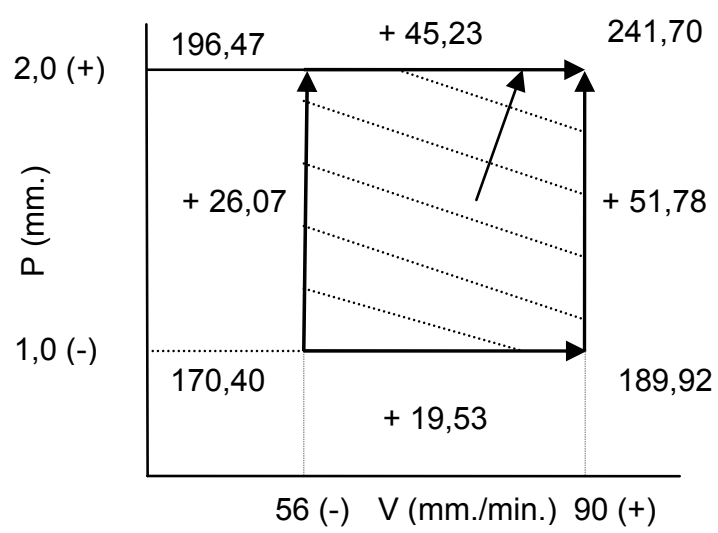

Fig. 1: Diagrama de dos vías, profundidad de corte $(\mathrm{P})$ vs. velocidad de avance $(\mathrm{V})$

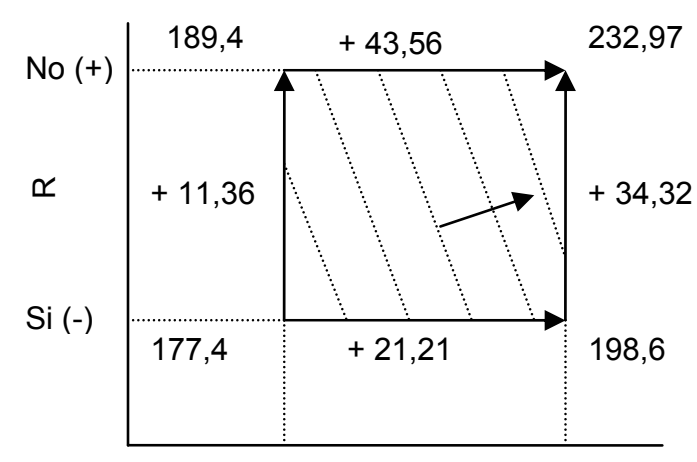

$56(-) \quad \vee(\mathrm{mm} . / \mathrm{min}) .90(+)$

Fig. 2: Diagrama de dos vías, refrigerante $(\mathrm{R})$ vs. velocidad de avance (V)

Potencia $=192.78+11.56 \mathrm{X}_{2}+16.19 \mathrm{X}_{3}$

Autores como Schmid (2005) define la potencia teórica necesaria para realizar el trabajo de corte como una función asociada a la resistencia al corte, espesor (profundidad de corte), ancho de corte, número de dientes de la fresa, avance por diente y la velocidad de giro de la herramienta en rpm, sin considerar, el efecto de la lubricación sobre el corte, lo cual deja un vacío que es cubierto con esta experimentación.

Sin embargo, se puede apreciar que la potencia consumida es directamente proporcional a la velocidad de avance, tal y como los valores de potencia consumida experimentalmente hacen ver, (Liu y Li, 200la; Engqvist et al., 1999), ya que el efecto antifricción de las herramientas de carburo de tungsteno favorece este comportamiento indistintamente al uso de fluido de corte.

La potencia teórica, depende del avance por diente, la velocidad de corte y el ancho de corte sin considerar la profundidad de corte $y$ el uso de refrigerante, Barbashov (1981) define una ecuación para el consumo de potencia con incidencia de la velocidad de avance y (Burghardt et al, 1965) señala que el avance y la profundidad de corte son proporcionales a la fuerza de corte aplicada, que esta igualmente en concordancia con los últimos experimentos planteados por Jensen y Shin (1999) parte 1 y 2.

No se puede considerar que el método más eficiente de maquinado es cuando se usan los mayores avances y profundidades de corte posibles, donde se considera la eficiencia del proceso como el consumo de potencia por unidad de volumen de viruta producida, sin tomar en cuenta el consumo energético para lograrlo.

\section{CONCLUSIONES}

Se logra el menor consumo de potencia con el uso de refrigerante y se comprueba cuando se trabja con una disminución en la velocidad de avance de 90 a $56 \mathrm{~mm} . / \mathrm{min}$.

Se demuestra, que la velocidad de avance es la variable de mayor significancia asociada con el refrigerante; en la medida en que la velocidad decrece y se usa refrigerante disminuye el consumo de potencia drásticamente, generando un decrecimiento de 55,53 W $(28,84 \%)$ en relación con el punto de operación más desfavorable. La velocidad de avance asociada a la profundidad disminuye el consumo de potencia en un $29,5 \%$ y la interacción de estas dos variables produce un consumo de potencia aun más bajo con baja velocidad de avance y profundidad. 
La utilización de esta nueva herramienta computacional SEMPRO 2, permitió comprobar la validez de los resultados, los valores de significancia de las variables estudiadas y las ecuaciones planares de primer orden en la superficie de respuesta de la zona estudiada.

\section{AGRADECIMIENTOS}

Al Postgrado en Ingeniería de Mantenimiento y al Laboratorio de Instrumentación Científica LIC de la Facultad de Ciencias, de la Universidad de Los Andes por su colaboración para esta investigación.

\section{REFERENCIAS}

Aoyama, H. y A. Ameraiya, Basic study on development of sensor to detect cutting force components based on Villari effect. Trans. of NAMRI/ASME (v28), 323-328 (2000).

Chern, G.L. y D.A. Dornfeld, Burr/breakout model development and experimental verification. Trans, of ASME, Journal of Engg. Materials and Technology (v118, n2), 201-206 (1996).

Engqvist, H., S. Ederyd, N. Axen, y S. Hogmark, Grooving wear of single-crystal tungsten carbide. Wear (v230), 165-174 (1999).

Jensen S.A. y Y.C. Shin., Stability Analysis in Face Milling Operations, Part 1: Theory of Stability, Journal of Manufacturing Science and Engineering Published by The American Society of Mechanical Engineers. 600-605 (1999).

Jensen S.A. y Y.C. Shin., Stability Analysis in Face Milling Operations, Part 2: Theory of Stability, Journal of Manufacturing Science and Engineering Published by The American Society of Mechanical Engineers, 606-614 (1999).

Liu, K. y X.P. Li, "Ductile cutting of tungsten carbide." Journal of Materials Processing Technology (vll3), pp 348-354 (2001).
Barbashov, F., "Manual del Fresador". Editorial Mir, 211-214, Moscu, Rusia (1981).

Box, G.E.P., W.G. Hunter y J.S. Hunter, "Statistics for Experiments", $3^{\mathrm{a}}$ ed., John Wiley \& Sons, Inc., USA. New York (1978).

Burghardt, H., A. Axelrod, y J. Anderson, "Manejo de las Máquina Herramientas", Editorial McGraw Hill, pp 217, Madrid, España (1965).

Casillas, A., "Máquinas". Graficas Reunidas. $5^{\mathrm{a}}$ ed., Madrid, España (1982).

Gutierrez, F. y R. De la Vara, "Análisis y Diseño de Experimentos", Mc Garw Hill, $1^{\text {a }}$ ed, 237-264. Cd. México. México (2003).

Krar, S. y A. Check, "Tecnología de las Máquinas - Herramientas", Alphaomega Grupo Editor. $5^{\mathrm{a}}$ ed., México, México (2002).

Montgomery, D., "Introduction to Satistical Quality Control", $3^{\text {rd }}$ ed., Jhon Wiley \& Sons, Inc., New York. (1996).

Chu, C.H. "A framework for burr minimization in a CAD/CAM integrated environment." Research Report. Berkeley, CA: Laboratory for Mfg. Automation, Univ. of California at Berkeley. (1999).

Sarache L. y M. Peña, "Software para el uso del Diseño Multifactorial de Dos Niveles en la Investigación SEMPRO 2". $5^{\text {to. }}$ Congreso Nacional de Ingeniería Mecánica, Mérida, Venezuela (2004).

Sarache, L., "Diseño de Experimentos $y$ Análisis Multifactorial Aplicado a la Industria". Facultad de Ingeniería, Universidad de Los Andes. Mérida. Venezuela. 585-590 (2001).

Schmid, L: http://fai.unne.edu.ar/contenido lapuntes_de_TM.htm (fecha de la consulta Mayo $18, \overline{(2005)}$. 